\title{
Foundation Course for Undergraduate Medical Education Program: A Stitch in Time
}

\author{
R Chaturvedi ${ }^{1}$, Rajshree R Gupta ${ }^{2}$ \\ Keywords: Communication skills, Ethics, Professionalism. \\ Journal of Medical Academics (2019): 10.5005/jp-journals-10070-0040
}

Medical profession has been regarded as a noble profession since time immemorial. A gap has however developed over the years between society's expectations from medical professionals and delivery of health care by them. This has not only dented the reputation of the doctor but also maligned the profession. This has resulted in various accusations that have been made against doctors in recent past.

It was as early as the 137 years back that a wake-up call was given by the Journal of the American Medical Association (JAMA), when it wrote in 1892:

"No physician, however conscientious or careful, can tell what day or hour he may not be the object of some undeserved attack, malicious accusation, black mail or suit for damages..."

Some factors that lead to such a state could be poor training of medical professionals, communication skills, grievance redressal system, high workload of hospitals, literacy, and political interference, to name a few. ${ }^{1}$ While all these factors might be beyond the control of medical education systems, the first two can be mended through intervention of the medical colleges. The first is poor training, as medical education is being criticized for its quality of education. ${ }^{2}$

The second factor that requires attention is poor communication skills. Poor communication skills can mar even a well-managed patient, especially in a country where illiteracy is still a problem. Likewise, good communication can cover-up relatively deranged state of affairs. This applies to all the health workers, medical or nonmedical. Hence, medical facilities with staff poorly equipped with adequate communication skills can run into difficulties.

Lieberman has identified three subjects on which the knowledge of medical profession is in general weak. They are manners, morals, and medicine. ${ }^{3}$ The medical education must address these issues. This is the need of the hour. The Medical Council of India (MCI) has identified these weak areas and has taken steps to make fundamental changes in the undergraduate medical curriculum. The aim is to incorporate the attributes of empathy, care, and communication skills in the fresh medical graduates through a specified course. This is proposed to be achieved through a dedicated one month exclusive "Foundation Course" spanning over 175 hours, at the beginning of the MBBS program. The course is implemented simultaneously by medical colleges all over India in the month of August every year, beginning 2019.

An orientation to the concept of professionalism and ethics is vital for a doctor. This component provides students with understanding that clinical competence, communication skills, and sound ethical principles are the foundation of professionalism. It also gives an understanding of the consequences of unethical and unprofessional behavior, value of honesty, integrity, and respect in

${ }^{1}$ Army College of Medical Sciences, Delhi Cantonment, Delhi, India
${ }^{2}$ Department of Physiology, Army College of Medical Sciences, Delhi
Cantonment, India

Corresponding Author: Rajshree R Gupta, Department of Physiology, Army College of Medical Sciences, Delhi Cantonment, India, Phone: +919013101527, e-mail: rajshreegupta2008@gmail.com

How to cite this article: Chaturvedi R, Gupta RR. Foundation Course for Undergraduate Medical Education Program: A Stitch in Time. J Med Acad 2019;2(2):37-38.

Source of support: Nil

Conflict of interest: None

all interactions. These areas were not touched upon previously in the curriculum. The students used to learn these values during the MBBS course by observing the senior doctors. Through foundation course, the $\mathrm{MCl}$ has ensured that these noble virtues of a doctor are understood at a very early stage of their career.

As has been appropriately put by Dr Hemant Deshmukh, dean of Seth GS Medical College, Mumbai, "For years, MBBS courses have started with anatomy lectures on day one. This is the first time that the entry level curriculum will be something different!" ${ }^{4}$ Earlier, the first year medical students were directly subjected to theory lectures of anatomy, physiology, and biochemistry. They were introduced to their teachers very formally either in the lecture halls or in practical labs. The implementation of the foundation course in the beginning of their career provides ample opportunities to know their prospective teachers through informal interactive sessions.

The one month-long course gives time for smooth transition for the students who are around 17 years of age, coming directly from school. The course also helps these youngsters understand various aspects about medical profession. The first year undergraduate students are also sensitized to various learning methodologies, which they might not have been exposed to till school, such as small group discussions, skills laboratory, simulations, documentation, and the concept of self-directed learning.

Keeping in mind their diverse backgrounds, in terms of culture, economy, geography, and medium of instruction in school, the course incorporates various soft skills (such as language, communication, etc.), sports, and extracurricular activities. The aim is to ensure that the students get an opportunity to appreciate and accommodate the similarities and differences and to feel at par with each other-and above all develop team spirit.

Since it is the first time that the course is being conducted, feedback from the students will go a long way in improving the course in future. 


\section{Conclusion}

Introduction of the foundation course by the $\mathrm{MCl}$ is a stitch in time. The foundation course would contribute to instill empathy and care in the Indian medical graduates. It is expected that the course is not merely conducted for a month and forgotten but the teaching of attitude, ethics, communication, and early clinical exposure continues. Questions related to these aspects have been incorporated in the internal assessments with a hope that these noble values are etched in the students' young minds to remain there forever. The responsibility now lies on the shoulders of the medical teachers and doctors to comprehend the ethos of the course and implement it effectively and efficiently. The teachers will have to raise themselves by another notch to be the role models for students. Teachers need to demonstrate these attributes in their daily conduct to pose as ideal examples to the young medical student. Though the introduction of this foundation course appears a small step, but it might prove to be a giant leap for the medical profession, in the years to come.

\section{References}

1. Ghosh K. Violence against doctors: a wake-up call. Ind J Med Res 2018;148(2):130-133. DOI: 10.4103/ijmr.IJMR_1299_17.

2. Royal KD, Rinaldo JCB. There's education, and then there's education in medicine. J Adv Med Educ Prof 2016;4(3):150-154.

3. Lieberman GF. Cited 2019 Dec 14. Available from: https://www. brainyquote.com/authors/gerald-f-lieberman-quotes.

4. Shelar J. Foundation course for MBBS students to be rolled out on August 1. The Hindu. [Newspaper on the Internet] 2019 July 27 [cited 2019 Dec 18]: Education: [About 2 p]. Available from: https://www. thehindu.com/education/foundation-course-for-mbbs-students-tobe-rolled-out-on-august-1/article28725942.ece. 